\title{
Emerging Optocoupler Issues with Energetic Particle-Induced Transients and Permanent Radiation Degradation
}

\author{
R.A. Reed ${ }^{1}$, P.W. Marshall ${ }^{2}$, A.H. Johnston ${ }^{3}$, J.L. Barth ${ }^{1}$, C.J. Marshall ${ }^{4,7}$, \\ K.A. LaBel ${ }^{1}$, M. D'Ordine ${ }^{5}$, H.S. Kim ${ }^{6}$, M.A. Carts ${ }^{2}$ \\ 1. NASA/GSFC, Code 562, Greenbelt, MD 20771 \\ 2. NRL/SFA, 1401 McCormick Dr., Largo, MD 20785 \\ 3. JPL, Pasadena, CA 91109 \\ 4. NRL, Code 6611, 4555 Overlook Ave S.W., Washington, DC 20375 \\ 5. Ball Aerospace, 1600 Commerce St., Boulder, CO 80301 \\ 6. Jackson and Tull Chartered Engineers, Washington, DC 20018 \\ 7. Currently at NASA/GSFC, Code 562, Greenbelt, MD 20771
}

\begin{abstract}
Radiation-induced permanent degradation and single event transient effects for optocouplers are discussed in this paper. These two effects are independent to the first order and will be addressed separately. Displacement damage-induced degradation of optocoupler current transfer ratio is reviewed. New data are presented that show the importance of application specific testing and that generalized quantification of optocoupler CTR degradation can lead to incorrect predictions of actual circuit performance in a radiation environment. Data are given for various circuit loading and drive current parameters. Previous work that introduces the idea that two mechanisms exist for inducing transients on the optocoupler output is discussed. New data are presented that extends the evidence of this dual mechanism hypothesis. In this work measurements show that single event transient cross sections and transient propagation varies with circuit filtering. Finally, we discuss utilization of the optocouplers in the space environment. New data are applied to two examples: one on permanent degradation and the other on single event transient rates in high bandwidth applications.
\end{abstract}

\section{INTRODUCTION}

Optocouplers provide DC isolation between circuit blocks which increases the reliability of space-born instruments. Optocouplers vary in design and functionality, both of which can impact its response to a radiation environment. Figure 1 depicts a typical optocoupler.

Optocouplers are generally constructed of two die, separated by an optically transparent but electrically isolated medium. Information is transferred by light generated by a light emitting diode (LED), and sensed by a photodetector. The detector is either a phototransistor or a photodiode. The reader should review [1] for a description of optocoupler construction techniques.

\section{A. Review of Permanent Degradation of Current Transfer Ratio}

Past studies show permanent degradation of an optocoupler's current transfer ratio (CTR) occurring when it is exposed to a radiation environment $[2,3,4]$. Current transfer ratio is the photodetector collector current divided by the LED drive current. Barnes et al. [4] explored radiation degradation for typical optoelectronic devices designed in the 1980's. Gamma ray and neutron degradation were included in their discussions. From that early work, it was learned that optocouplers containing amphoterically Si doped GaAs LEDs and $\mathrm{Si}$ phototransistors could be especially sensitive to displacement damage. Optocouplers manufactured with GaAsP LED and a Si photodiode performed better.

Recent studies show increased degradation of CTR for modern commercial-off-the-shelf (COTS) optocouplers [1,5]. Rax et al. [1] characterized two types of optocouplers for radiation damage by gamma rays and protons. Both types contained a Si phototransistor. One contained a Darlington phototransistor constructed as a sandwich structure with a GaAlP LED. The other optocoupler contained an AlGaAs side emitting LED. The AlGaAs doped devices degraded the most.

The exact mechanism for the poor performance of the class of devices that contained amphoterically doped LEDs is not known. However, studies [2,3,4] show that displacement damage plays an important role. The recent study [1] dissected the optocouplers and determined that CTR degradation due to displacement damage in the LEDs was the major contributor to the degradation of modern optocouplers.

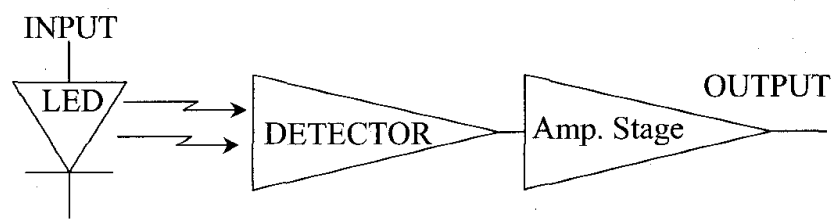

Figure 1. Typical Optocoupler design. 
The degradation of optocoupler CTR can cause spacecraft failures. In fact, the failure of the TOPEX/Poseidon spacecraft was attributed to the use of optocouplers manufactured with amphoterically doped. LEDs. These devices failed after the accumulation of $1.2 \times 10^{10}$ protons $/ \mathrm{cm}^{2}$ [1].

\section{B. Review of Single Event Effects}

Optocouplers are also susceptible to temporary radiation induced effects called single event transients (SETs). An SET occurs when charge freed by ionizing radiation is amplified and appears on the output of the optocoupler as a voltage pulse. LaBel et al. [6] presented data on optocouplers from three manufactures showing proton-induced voltage pulses on the output of the high bandwidth optocouplers, but no transients were observed on low bandwidth devices. The analysis suggested a combination of indirect and direct ionization based proton-induced transient effects. These transients are analogous to single event transients observed in linear devices [see for example 7,8].

The result of using the high bandwidth devices to isolate circuits that must function in a radiation environment can be a SET on the output of the optocoupler. If the SET propagates through follow-on circuitry, then system operation maybe impacted. A description of the effects of transients on the Hubble Space Telescope (HST) is given in LaBel et al. [6].

\section{Current Issues}

The current understanding of displacement damage degradation of each component of an optocoupler will be reviewed. New CTR degradation data will be presented showing the impact of using non-application specific radiation test data. New SET test data will be presented that supports the assumption about the mechanisms for inducing a transient presented in [6]. SET mitigation technique using circuit filtering is validated showing that application specific testing should be required. In the final section of this paper we will give two examples of using optocouplers in the space environment. We also discuss methodologies used to predict optocoupler performance in the space radiation environment.

\section{FACILITIES}

The $63 \mathrm{MeV}$ proton measurements were performed at the Crocker Nuclear Laboratory (CNL) cyclotron located at the University of California at Davis where the dosimetry accuracy is know to be about $10 \%$. The $195 \mathrm{MeV}$ proton exposures were carried out at the Indiana University Cyclotron Facility (IUCF) where dosimetry accuracy is known to be about $10 \%$. Loma Linda University Medical Center (LLUMC) synchrotron facility was used to perform $53 \mathrm{MeV}$ proton irradiations. The dosimetry is believed to be accurate to within 20\%. TRI-University Meson Facility (TRIUMF) at the University of British Columbia provided protons with energies between 65 and $225 \mathrm{MeV}$. Both ion chamber and plastic scintillator telescopes were used to measure the proton fluences, and the dosimetry is good to about $10 \%$. The National Superconducting Cyclotron Laboratory (NSCL) located at Michigan State University was used for the 240

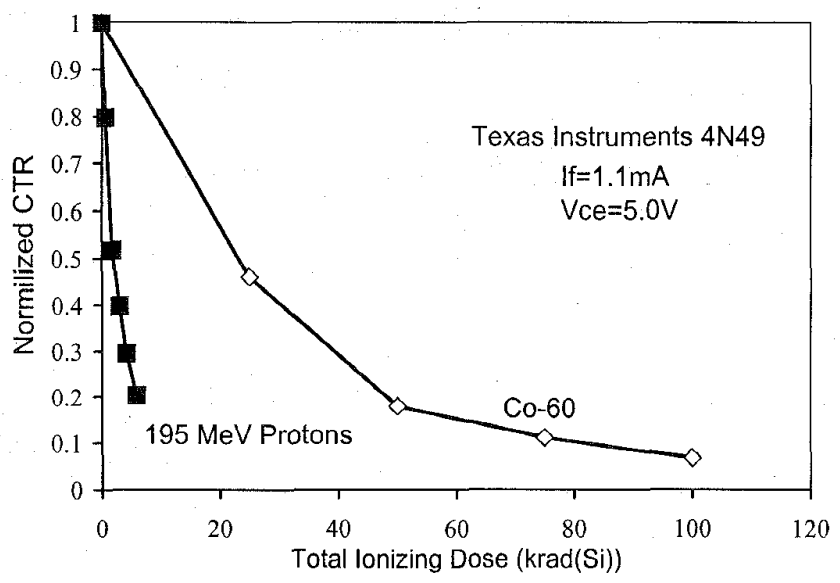

Figure 2 Gamma and $195 \mathrm{MeV}$ proton irradiations of Texas Instruments $4 \mathrm{~N} 49$ showing proton damage enhancement.

$\mathrm{MeV} \mathrm{He}\left(0.064 \mathrm{MeV}-\mathrm{cm}^{2} / \mathrm{mg}\right)$ measurements. Dosimetry was performed using a real time parallel plate avalanche counter (PPAC). The measured fluences are expected to be accurate to within $30 \%$.

\section{Permanent Radiation Damage}

Components contained in optocouplers can show performance degradation when exposed to certain radiation environments. The exact mechanism for degradation depends on the details of the type of optocoupler, the circuit in which it is used, and the radiation environment to which it is exposed. For example, Figure 2 compares $195 \mathrm{MeV}$ proton exposures of a Texas Instruments (TI) 4 N49 to that for gamma ray exposures. Proton irradiations cause significantly larger degradation than gamma ray exposures at equivalent doses. This type of optocoupler response is due to the greater amount of displacement damage for proton over gamma exposures [1]. Figure 2 shows that one must use the appropriate combination of degradation information to predict accurate performance in a mixed radiation environment.

\section{A. Displacement Damage Degradation Mechanism}

Optocoupler CTR can be degraded by displacement damage or TID effects. Displacement damage can affect the overall CTR of optocouplers by three different mechanisms: (1) degrading the lifetime in the photodetector, thereby reducing responsivity, (2) reducing the gain of the transistor used in basic types of optocouplers, and (3) reducing the light output of the LED. All three mechanisms have been observed, but mechanisms (1) and (3) are the most significant.

TID can degrade the light output of LED, the light transmitted by coupling medium, and/or the conversion of light to electrical signal by the photodector $[1,4]$. In this section we will focus on displacement damage effects with warning that for certain optocouplers TID effects can be the dominate mechanism.

1) Silicon Responsivity. The effect of lifetime damage on silicon responsivity depends on the light wavelength as well as on the underlying construction of the photodetector. For 
example, the absorption coefficient, $\alpha$ (distance required to decrease the intensity by a factor of $1 /$ e compared to the value at the surface) of two commonly used wavelengths are as follows:

$$
\begin{array}{ll}
\lambda=700 \mathrm{~nm}, & 1 / \alpha=8.5 \mu \mathrm{m} \\
\lambda=890 \mathrm{~nm}, & 1 / \alpha=40 \mu \mathrm{m}
\end{array}
$$

Most of the light is collected by diffusion, with significant light collection over a distance of approximately $3 \alpha$, unless the underlying region is physically limited to thinner dimensions. The minority carrier diffusion length, $\mathrm{L}$, is given by

$$
\mathrm{L}=[\mathrm{D} \tau]^{1 / 2}
$$

where $\mathrm{D}$ is the diffusion constant and $\tau$ is the minority carrier lifetime. The photoresponse will begin to be affected when the lifetime is reduced to the point where $L<3 \alpha$. Displacement damage reduces the minority carrier lifetime by the equation

$$
\Delta(1 / \tau)=\Phi / K_{1}
$$

where $\Phi$ is the proton fluence in $\mathrm{p} / \mathrm{cm}^{2} ; \mathrm{K}_{1}$ is the lifetime damage constant in $\mathrm{p}-\mathrm{sec} / \mathrm{cm}^{2}$; and $\Delta(1 / \tau)=1 / \tau-1 / \tau_{0}$ is the change in reciprocal lifetime after irradiation. For the two wavelengths considered above, the $50 \mathrm{MeV}$ fluence required to reduce the photoresponse by $20 \%$ is then $3 \times 10^{10} \mathrm{p} / \mathrm{cm}^{2}$ for a wavelength of $890 \mathrm{~nm}$, and $6.5 \times 10^{10} \mathrm{p} / \mathrm{cm}^{2}$ at $700 \mathrm{~nm}$. This calculation assumes a lightly doped p-substrate for both cases, and does not take into account the fraction of the light that is collected in the depletion region. This example does not apply to the HP devices discussed later in this paper and in [6]. They contain a photodiode fabricated monolithically in HP's bipolar IC process. The responsivity is on the order of $30 \%$, the junction region is on the order of 2 microns, and there is no lightly doped $p$ substrate [6].

2. Phototransistor Gain Degradation. Phototransistor gain is also affected by displacement damage, although this is only a factor for basic optocouplers that use simple one or twotransistor amplification stages such as the $4 \mathrm{~N} 49$ and $6 \mathrm{~N} 140$. The Darlington configuration further reduces the sensitivity of the $6 \mathrm{~N} 140$ to transistor gain degradation [6] (the best performance observed to date is on the HP $6 \mathrm{~N} 134$ which contains a photodiode). Gain degradation can be described by the equation

$$
\Delta\left(1 / \mathrm{h}_{\mathrm{FE}}\right)=\mathrm{K}_{2} *\left(2 \pi \mathrm{f}_{\mathrm{T}} \Phi\right)^{-1}
$$

where $\Delta\left(1 / h_{\mathrm{FE}}\right)$ is the change in the reciprocal of the commonemitter current gain, $\mathrm{h}_{\mathrm{FE}} ; \mathrm{K}_{2}$ is the lifetime damage constant similar to that discussed earlier; and $f_{T}$ is the gain-bandwidth product. Typical phototransistors have $f_{T}$ values of approximately $300 \mathrm{MHz}$, resulting in degradation of only a few percent at the fluence levels where significant changes occur in photoresponse. Thus, gain degradation is generally negligible compared to the other terms.

3. LED Degradation. Displacement damage studies on lightemitting diodes in the early $1980^{\prime}$ s showed that LED degradation could be described by a model in which radiationinduced recombination centers decreased the minority carrier lifetime. Consequently, the relative fraction of the injected current that went into non-radiative recombination is increased [4]. The earlier work showed that amphoterically Si-doped LEDs (these are also GaAs-based) were far more sensitive to proton displacement damage than either GaAs or AlGaAs LEDs. The AlGaAs device in the earlier work had a wavelength of $820 \mathrm{~nm}$. Damage constants for the amphoterically doped devices were about 300 times larger than for the other LED technologies.

More recent work [1] on AlGaAs LEDs with longer wavelengths $(880 \mathrm{~nm})$ showed that far more degradation occurred than indicated by the early study. The reason for this has not been established, but it may be due to improvements in LED fabrication technology that increase efficiency. Damage constants for the new technologies are of the same order of magnitude as the amphoterically doped devices used in the earlier work $[1,4]$. GaAsP devices (700 $\mathrm{nm}$ wavelength) have much lower damage constants. Degradation of AlGaAs LEDs is so severe that optocouplers using that particular technology are severely degraded at proton fluences of $2 \times 10^{10} \mathrm{p} / \mathrm{cm}^{2}$, dominating the performance of optocouplers that use them. Optocouplers with GaAlP LEDs experience only slight LED degradation, and those devices are dominated by the photoresponse of the detector. The result is that optocouplers at $700 \mathrm{~nm}$ are about two-orders of magnitude more tolerant than devices operating at $880 \mathrm{~nm}$. [1,4]

\section{B. Displacement Damage Degradation Testing and Predictions}

The most straightforward way to evaluate displacement damage is by irradiation with neutrons, using a high neutronto-gamma ratio in order to minimize ionization damage. However, the equivalence between neutron and proton damage is different for silicon and III-V devices, so one must know how the various mechanisms contribute to the overall degradation of CTR in order to establish the correct equivalence between the different environments.

Proton tests can be done in a relatively straightforward manner, but the displacement damage is strongly affected by proton energy. Ionization damage from protons will also contribute to degradation in the photodetector and transistor, further complicating the interpretation of test results.

Whether neutron or proton testing is selected for evaluation of CTR degradation by displacement damage, accurate space flight predictions rely on the knowledge of how the optocoupler degradation varies with particle energy. In the absence of measured CTR data, nonionizing energy loss (NIEL) curves must be known over proton energy if proton testing is selected. For neutron testing one must know not only NIEL over proton energies but also NIEL over neutron energies and the relationship between protons and neutrons. Truly accurate predictions require NIEL spectra for each semiconductor used in manufacturing the optocoupler and knowledge of how each component degrades to displacement damage.

For purposes of quantifying displacement damage from combinations of particles across various energy ranges, NIEL 


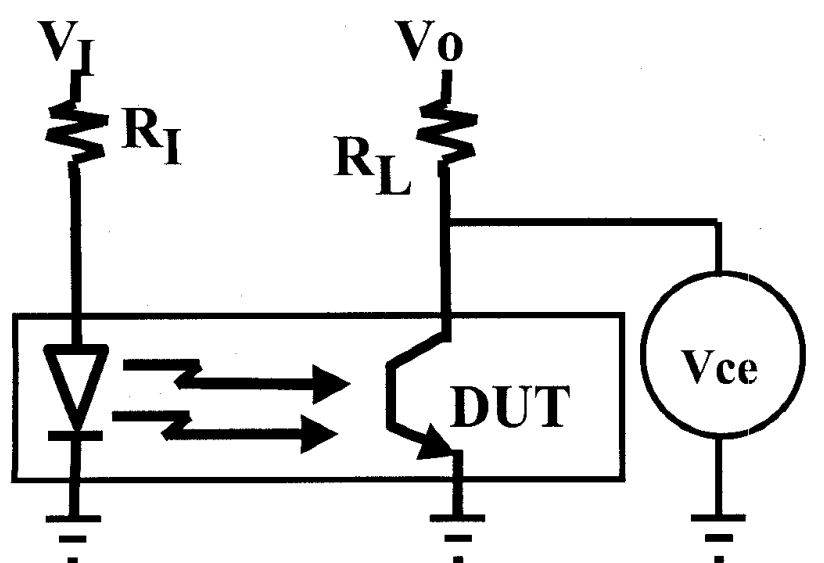

Figure 3. Schematic shown experimental setup for measure CTR degradation.

plays the same role as linear energy transfer for the case of ionizing radiations. By knowing the NIEL rate for each particle and energy in question (in the material of interest), it becomes possible to combine the damaging effects of all particles by integrating over their energy range. The resulting linear combination represents the displacement damage dose. This dose has been shown to be a useful metric for certain materials on some systems $[9,10]$. However, in GaAs there remain outstanding questions regarding the validity of this approach. Discussions of some of the device and material specific issues are provided [11,12].

Non-application specific displacement damage characterization of CTR degradation given in some archival databases and journals are typically performed by holding the phototransistor collector-emitter voltage $\left(\mathrm{V}_{\mathrm{ce}}\right)$ constant while measuring the CTR. Data presented in the next sub-sections will show that utilization of data collected with constant $V_{c e}$ may lead to incorrect prediction of the actual degradation for certain applications.

1) Experimental Test Setup, Facility and Pre-irradiation Values. Figure 3 shows the experimental setup. Two HP high precision voltmeters were used to monitor the voltage drop across $R_{I}$ and $R_{L}$. Two independent power supplies were used to sweep $V_{I}$ and $V_{O}$. Sweeping $V_{I}$ causes the LED drive current $\left(I_{f}\right)$ to change values. The drive current is computed from the ratio of the voltage drop across $R_{I}$ to the resistance $\left(R_{I}\right)$. Sweeping $V_{O}$ causes the phototransistor $V_{c e}$ to change values. The collector-emitter voltage is the difference between $V_{O}$ and the measured voltage drop across $R_{L}$. The collector current $\left(\mathrm{I}_{\mathrm{C}}\right)$ is computed from the ratio of the voltage across $R_{L}$ to the resistor value $\left(R_{L}\right)$. The optocouplers used in this portion of the study were Texas Instruments $4 \mathrm{~N} 49$. It contains a side emitting LED and a phototransistor. IUCF was the facility used to conduct the irradiations.

The pre-irradiation sweeps are shown in Figure 4. The collector current $\left(\mathrm{I}_{\mathrm{c}}\right)$ is plotted as a function of the forward current $\left(I_{f}\right)$ for various collector-emitter voltages $\left(V_{c c}\right)$. This characterization is analogous to a simple $I-V$ curves for a transistor. Notice that $I_{c}$ increases proportionally with $I_{f}$ with a slope governed by the current gain (analogous to the active

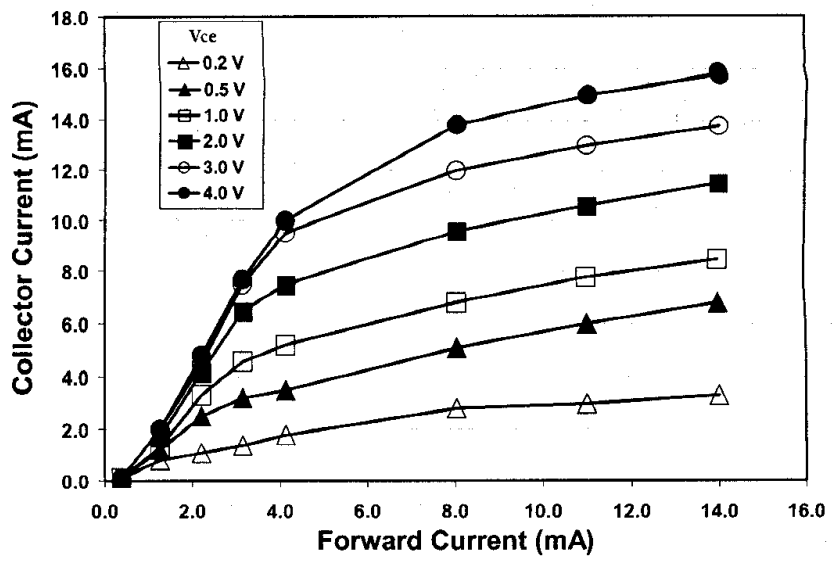

Figure 4. Pre-irradiation I-V characteristics of a TI 4N49. Radiation damage changes the shapes of these curves.

region for simple transistors). When the optocoupler is fully on, this proportionality breaks down, and the curves for various $V_{c e}$ values flatten out at nearly constant values of $I_{c}$. Further increases in $I_{f}$ result in very small increases in $I_{c}$ (analogous to the saturation region for simple transistors).

As was described in the previous section, displacement damage can degrade each component of an optocoupler. The impact of this degradation is seen in how the shapes of the curves shown in Figure 4 change with radiation damage. These shapes of the curves can change when the optocoupler is exposed to either an environment that induces displacement damage or deposits total ionizing dose. If $\mathrm{V}_{\mathrm{ce}}$ is held at a constant value during testing, then the response of the device to radiation will be different than the case where $V_{c e}$ is changing due to radiation-induced effects.

2) Experimental Results and Discussion. Consider the following thought experiment involving two forward currents: $\mathrm{I}_{\mathrm{f}}=8 \mathrm{~mA}$ (analogous to saturation region for simple transistor) and $\mathrm{I}_{\mathrm{f}}=1 \mathrm{~mA}$ (analogous to active region of simple transistor). Further assume that the major degradation mechanism is LED light output degradation (believed to be the dominate mechanism for this type of optocoupler). Light output degradation of the LED is analogous to a reduced base current in a simple transistor. Reduced light output due to radiation can also be viewed as having the same effect as reducing the forward current in an unirradiated device, i.e. degraded light output due to radiation or reduced drive current will both reduce $I_{C}$. If the forward current is changed while the device is in saturation, the collector current, and hence the CTR, will remain essentially constant. However, if the forward current is changed when operating in the active region then the optocoupler $I_{C}$ will change. It is easy to see from this thought experiment that different values for $V_{c e}$ will give different radiation responses.

The data represented by solid filled symbols connected by solid lines in Figure 5 show a comparison of the CTR degradation at two different loads $\left(R_{L}\right)$ for the same device. $\mathrm{V}_{\mathrm{O}}$ was set so the initial $\mathrm{V}_{\mathrm{ce}}=0.3 \mathrm{~V}$ for both loads. The test conditions that existed during the $2.7 \mathrm{ohm}$ measurements are consistent with holding $\mathrm{V}_{\mathrm{ce}}$ constant at $0.3 \mathrm{~V}$. We note that the 


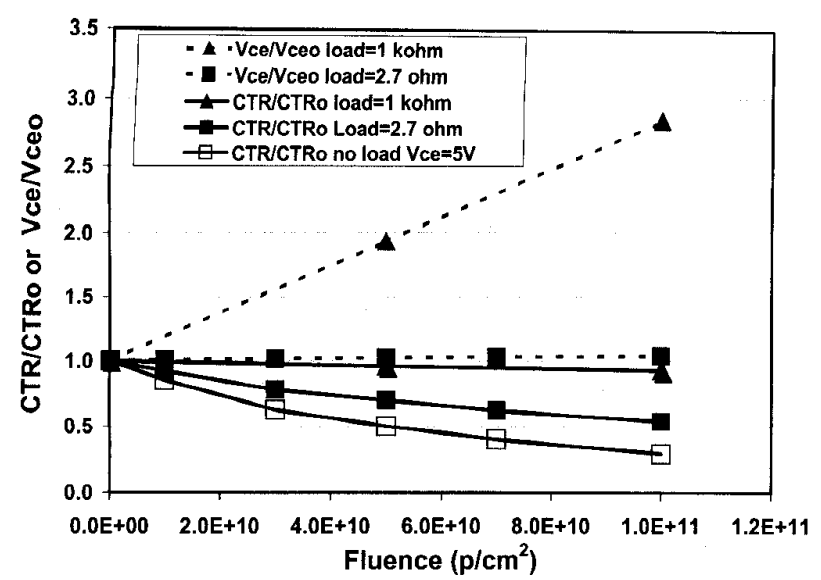

Figure 5. Plot of either normalized CTR (solid lines) or normalized Vce (dashed lines) for a $4 \mathrm{~mA}$ forward current. This shows the load dependence on the radiation response. It also demonstrates that testing at a fixed Vce would significantly over predict the CTR degradation.

CTR for the case where the load was $2.7 \mathrm{ohm}$ degraded by almost $50 \%$ of its original value after an exposure of $1 \times 10^{11}$ $\mathrm{p} / \mathrm{cm}^{2}$ while the $1 \mathrm{kohm}$ load degradation was $92 \%$ of the original value. This is nearly a factor of two difference for the same initial conditions.

The dashed lines in the figure show the radiation-induced change of $V_{c e}$ with fluence for each load. For the $2.7 \mathrm{ohm}$ load case $V_{c e}$ is remained constant at $0.3 \mathrm{~V}$. In the case where the load is $1 \mathrm{kohm}, V_{\text {ce }}$ increased with increasing fluence. Radiation degraded the output of the LED, as evident in the $2.7 \mathrm{ohm}$ loading, but for the $1 \mathrm{kohm}$ loaded case output degradations was compensated for by an increase the emitter-collector voltage. These data clearly show that a significantly inaccurate prediction of CTR degradation would occur if application specific testing was not carefully done for this type of application.

The data represented by the unfilled squares in Figure 5 show the CTR degradation for a fixed $\mathrm{V}_{\text {ce }}$ of $5 \mathrm{~V}$. The last data point on this curve shows a degradation of $29 \%$ of the original value. Compare this to the $92 \%$ when $V_{c e}$ was allowed to change (1 kohm load). Data collected at $V_{c e}=5 \mathrm{~V}$ over predicts the degradation in the $1 \mathrm{kohm}$ load case by a factor of 4.6 .

These data are clear evidence that, in order to accurately characterize the radiation degradation of CTR for optocoupler operating in the saturation region, one should test the devices using the exact application parameters. Several publicly accessible databases and journals give data collected at fixed $\mathrm{V}_{\text {ce. }}$. These data can be used to obtain worst case estimates of degradation. Note that this technique could significantly over predict the degradation.

One suggestion to mitigate the effects of radiation degradation of CTR is simply to use the highest drive current possible while minimizing $\mathrm{V}_{\mathrm{ce}}$ for the desired collector current. This will drive the optocoupler into saturation. For example, comparing the data in Figure 4 , if the desired collector current is $4 \mathrm{~mA}$ the best selection of $\mathrm{I}_{\mathrm{f}}$ and $\mathrm{V}_{\mathrm{ce}}$ would be $5 \mathrm{~mA}$ and $0.5 \mathrm{~V}$. If an $\mathrm{I}_{\mathrm{f}}$ of $2 \mathrm{~mA}$ and $V_{c e}$ of $4 \mathrm{~V}$ had been

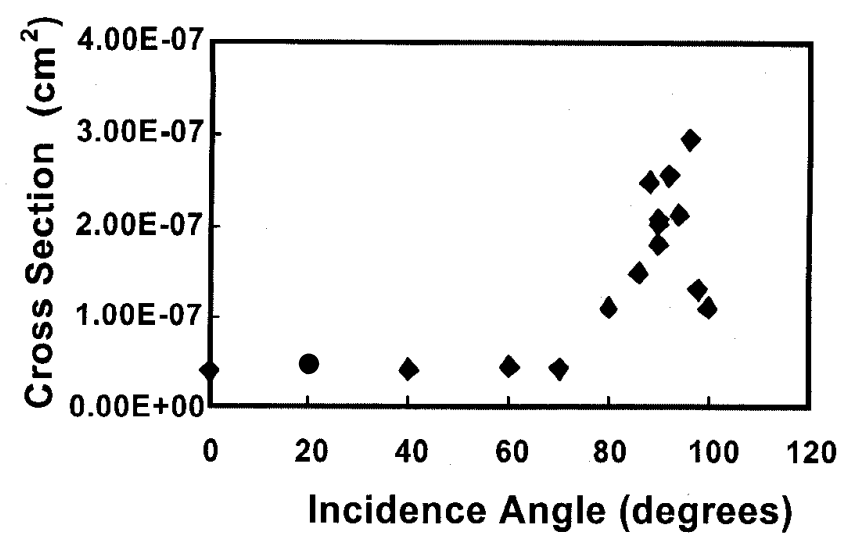

Figure 6. The order of magnitude increase in proton transient crosssection at grazing incidence is evidence of the direct ionization mechanism in the optocoupler photodiode [6].

chosen then the device would be more susceptible to changes in LED output. Care should be given to reliability concerns when operating optocouplers in this mode $[1,6]$.

\section{SingLe EVENT TRANSIENTS}

Several studies of single ionizing particle irradiations of optoelectronic devices have been published. Most of these studies focus on devices used in box-to-box communication links. It has been shown that a single ionizing particle passing through an optoelectronic device will free enough charge in the detector's depletion region to produce a voltage pulse on the electrical output. The pulse is classified as a single event transient (SET). These devices require a very small amount of charge to be freed to produce a change in the electrical output. In fact, the charge is so small that ionizations from a single proton can produce enough charge to change the state of the output. Several studies have concluded that direct ionization from protons will dominate the single event error rates in orbital proton environments.

In this section we examine proton-induced single event transients in high bandwidth optocouplers. This phenomenon was first reported by LaBel, et al., in [6]. Figure 6, taken from that work, reveals the $63 \mathrm{MeV}$ proton cross sections at varying angles for transients reported in that study. The key findings, in addition to the existence of direct ionization based protoninduced transient effects, were: high bandwidth devices appear to be the most susceptible, transients may persist on the output for $>100 \mathrm{~ns}$ (this period seems to be determined by the bandwidth of the device), transients appear to be initiated in the photodiode receiver internal to the coupler, and it appears that transients can be initiated by direct ionization by protons at grazing angles of incidence onto the photodiode. In addition, it was suggested that filters implemented at the coupler output could prevent the transient from propagating to the follow-on circuitry. In this study, we further examine the possibility of direct ionization induced transients, and we report new measurements describing the efficacy of filterbased suppression circuitry.

We report new transient test data on two HewlettPackard versions of the 6N134, the HCPL-6631 and 6651 . 


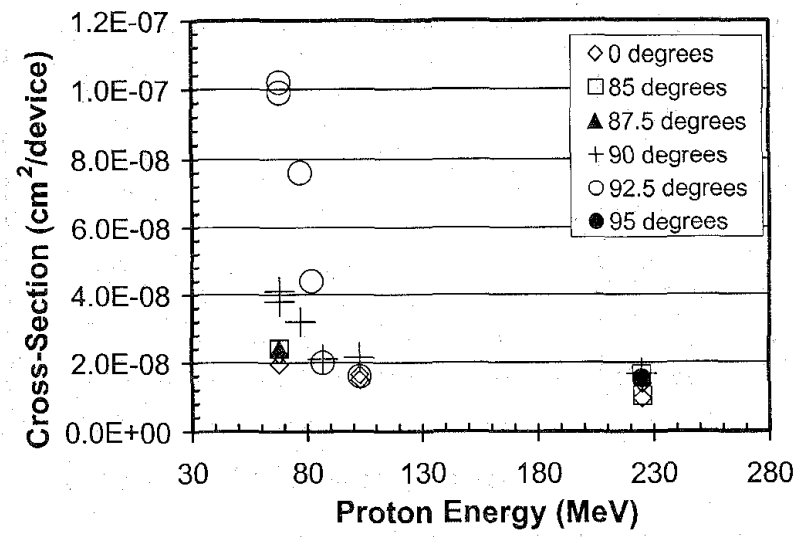

Figure 7. Proton-induced cross-section measurements at various proton energies and angles of incidence. The transient cross section for normal incidence does not vary strongly with energy above 60 $\mathrm{MeV}$, but the enhanced sensitivity to grazing angle trajectories is only seen at the lower proton energies.

These are multi-channel hermetically sealed, high speed (10 MHz), high CMR (common mode (noise) rejection) TTL logic level output optocoupler in a ceramic LCC (leadless chip carrier). Data from this same device type are shown in Figure 6. Each channel contains a GaAsP LED, which is optically coupled to a photodiode integrated with TTL level output circuitry. All Hewlett-Packard devices within this family use the same dies, and these tests are applicable to the whole family.

\section{A. SET Mechanism}

These data of Figure 7 indicate an almost constant cross section over the energy range from $68 \mathrm{MeV}$ to $225 \mathrm{MeV}$ for normally incident protons. Interestingly, there is a dramatic difference in the angular dependence between the higher and lower proton energies. The lower energy shows an increased cross section at near grazing incidence angles, similar to that measured on the same device and shown in Figure 6. This dependence is taken to be evidence of events initiated by direct ionization by protons traversing across the diameter of the coupler's internal receiver photodiode. At the $225 \mathrm{MeV}$ energy point, the enhancement at high angles is not detectable. We note the $\sim 42 \%$ decrease in $-\mathrm{dE} / \mathrm{dX}$ at the higher energy (from 0.0082 to $0.0035 \mathrm{MeV}-\mathrm{cm}^{2} / \mathrm{mg}$ ) and suggest that at the $225 \mathrm{MeV}$ energy the direct ionization mechanism is no longer important.

The same test setup and devices were taken to MSU. Data where collected at NSCL using the $240 \mathrm{MeV} \mathrm{He}$ ions. Transients were observed at several angles of incidence up to 85 degrees. The transients are most certainly due to direct ionization. These data indicate that the threshold LET for near grazing angles is below $0.064 \mathrm{MeV}-\mathrm{cm}^{2} / \mathrm{mg}$. This is consistent with the energy dependence seen in the proton data of Figure 7.

An analysis of the typical operation of the HP6651 optocoupler was based on the internal components. Optoelectronic properties and geometric assumptions were given in ref [6]. This analysis showed that light from the LED typically generates $6.3 \times 10^{5}$ ion pairs $(101 \mathrm{fC})$ in the detector

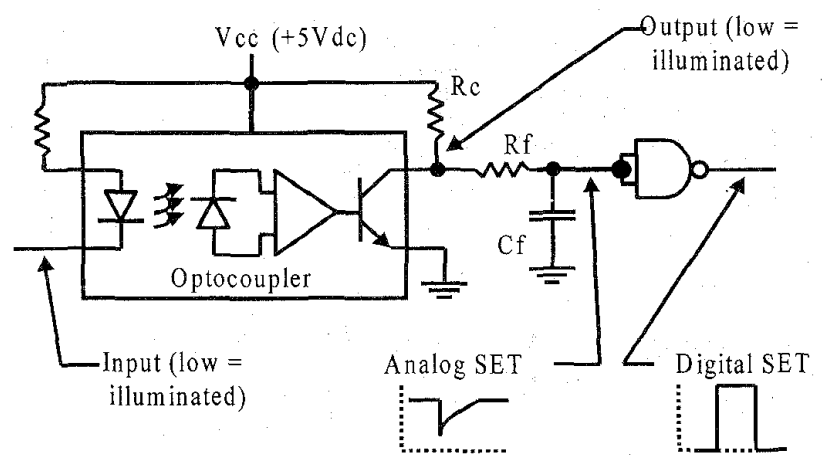

Figure 8. Schematic representation of transient test circuit showing the filter network and the two probe locations for analog and digital transient capture.

to produce a signal on the output of the optocoupler. Note that this is not a threshold, but a typical value. The threshold (and corresponding critical charge) must be lower than this typical value. Following this analysis, we determined that the critical charge for initiating transients must be less than that deposited by a $35 \mathrm{MeV}$ proton traversing the diameter of the diode. This agrees with the data above and is compelling evidence for the role of the direct ionization mechanism for grazing angle events.

\section{B. SET Mitigation}

Additional testing was performed at U.C. Davis Crocker Nuclear Laboratory using $63 \mathrm{MeV}$ protons. The test circuit is depicted in Figure 8 , which replicates a typical circuit implementation of the optocoupler for a satellite application. Using this circuit, we examined the possibility of transients in both the illuminated and in the unilluminated states. We saw no evidence that events could be initiated while the LED was active.

Measurements were conducted using an embedded National Instruments ${ }^{\text {B }}$ VXI controller running Labview ${ }^{(B)}$ software. The core of the test was a 5 Gsps (Giga samples per second) digital oscilloscope manufactured by Tektronix ${ }^{\circledR}$ (model TVS625) and its companion $1.5 \mathrm{GHz}$ bandwidth active probe (model P6245). Additional equipment consisted of a VXI digital I/O for controlling and monitoring the test board, GPIB power supply for the support circuitry as well as the DUT, and current monitoring.

A DC signal from the experiment controller is buffered and fed to the optocoupler. The optocoupler open-collector output is sampled by an oscilloscope with a high-speed, low capacitance active FET probe. It also feeds either an AC or ACT logic gate buffer (both being used in the application of interest). The transient monitored at the optocoupler output will exhibit the analog characteristics of the SET, but the buffered signal will generally be a clean TTL or $5 \mathrm{~V}$ CMOS level signal. The buffered signal is sampled by a passive oscilloscope probe. The buffered signal is fed to a conditional inverter along with the DC signal controlling the optocoupler input. Thus, the output of the conditional inverter is always nominally logic level LOW, with a SET always appearing here as a positive-going pulse. 


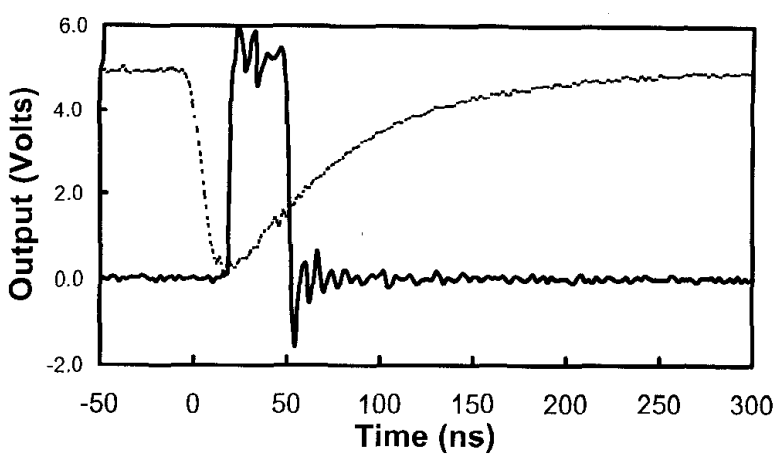

Figure 9. A typical transient from the HP6631 optocoupler as measured at the optocoupler output and at the circuit output as indicated in the previous figure. The filter values were: $\mathrm{Rc}=2.0$ $\mathrm{Kohm}, \mathrm{Rf}=0 \mathrm{ohm}$ and $\mathrm{Cf}=0 \mathrm{pf}$.

The optocoupler output stage is open collector, and the value of the collector resistor, $R_{c}$, affects the response of the circuit to SETs. The circuit response is additionally affected by capacitance on the node. Both of these values were made changeable and were test variables, as was the supply voltage to the optocoupler and the RC filter added to the circuitry to investigate mitigation techniques.

Figure 9 shows an example of a transient SET captured at the probe points indicated in Figure 8 . The digital output is initially low then is driven high for $\sim 50 \mathrm{~ns}$. Note the approximately exponential decay of the analog transient and also the $\sim 40$ ns delay between the onset of the analog transient and the digital transient. This $\sim 40$ ns period was constant across all transient events measured, and shorter transient events, as measured at the analog probe point, did not propagate to the next stage.

Table 1 illustrates the circuit response to varying resistor combinations in the passive filter circuit. The event cross sections and event durations at the output of the analog circuit reflect the ability of the filter to shorten the transient duration and reduce the total number of events reaching the circuit output. In all cases, the value of $\mathrm{C}_{\mathrm{f}}$ was $0 \mathrm{pf}$. For the cases shown, the more dramatic effect corresponds to the addition of a series resistance $\left(R_{f}\right)$. The resulting $R C$ network tends to suppress the transient propagation to the output, but it also compromises the circuit bandwidth, which in some applications may be unacceptable.

Table 1

Passive filtering of transient events impacts measured cross-section and duration.

\begin{tabular}{|c|c|c|c|c|c|c|}
\hline $\mathrm{R}_{\mathrm{C}} \Omega$ & $\mathrm{R}_{\mathrm{f}} \Omega$ & $\begin{array}{c}<20 \\
\mathrm{~ns}\end{array}$ & $\begin{array}{c}20- \\
40 \mathrm{~ns}\end{array}$ & $\begin{array}{c}40-60 \\
\mathrm{~ns}\end{array}$ & $\begin{array}{c}\text { Total } \\
\text { Events }\end{array}$ & $\begin{array}{c}\text { Cross } \\
\text { Section }\end{array}$ \\
\hline $5.11 \mathrm{~K}$ & 0 & 2 & 7 & 32 & 41 & $4.3 \mathrm{E}-08$ \\
\hline $2 \mathrm{~K}$ & 0 & 4 & 29 & 6 & 39 & $4.1 \mathrm{E}-08$ \\
\hline 511 & 0 & 7 & 23 & 2 & 32 & $3.4 \mathrm{E}-08$ \\
\hline $2 \mathrm{~K}$ & $2 \mathrm{~K}$ & 18 & 1 & 0 & 19 & $2.0 \mathrm{E}-08$ \\
\hline 511 & $2 \mathrm{~K}$ & 12 & 0 & 0 & 12 & $1.3 \mathrm{E}-08$ \\
\hline 511 & $5.11 \mathrm{~K}$ & 0 & 0 & 0 & 0 & $1.0 \mathrm{E}-10$ \\
\hline
\end{tabular}

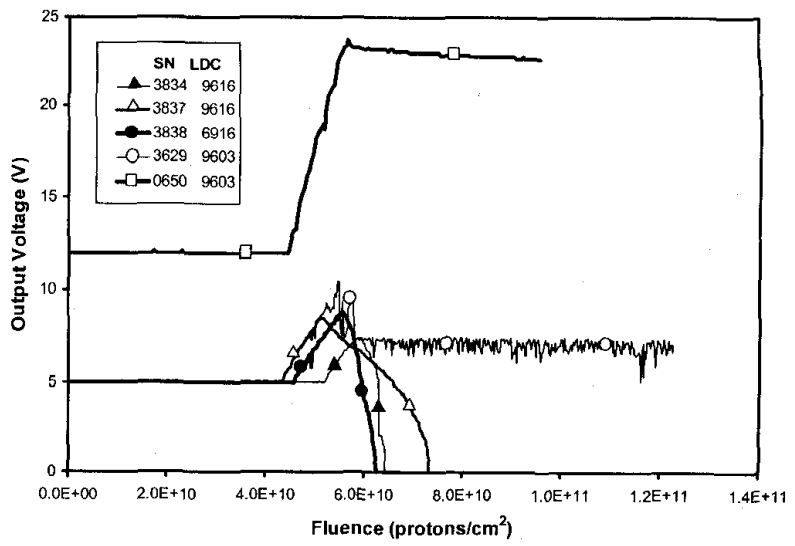

Figure 10. Proton irradiation of a DC/DC converter. It was shown in [13] that radiation-induced optocoupler degradation was the failure mechanism.

\section{USING OPTOCOUPLERS IN THE SPACE RADIATION ENVIRONMENT}

This section will give a two examples of how optocouplers are used in the space radiation environment and will present topics that should be given careful consideration when predicting optocoupler performance. The first example is a DC/DC converter, a hybrid that contains an optocoupler. The second is SET data collected on an instrument on the Hubble Space Telescope.

1) Using optocouplers that Show Displacement Damage Degradation in a Space Radiation Environment. Interpoint's $\mathrm{MHF}+2805 \mathrm{~S}$ and $\mathrm{MHF}+2812 \mathrm{~S}$ contain a Hamamatsu optocoupler. These hybrids were selected for use on a NASA spacecraft. The device was tested for Co-60 gamma irradiations and found to pass the mission requirements. Luckily for the program we found out by word of mouth that this hybrid contained an optocoupler that was known to fail at very low levels of proton fluence. Proton irradiations were carried out on a set of MHF+ devices. Figure 10 shows the how the output of the $5 \mathrm{~V}$ and $12 \mathrm{~V}$ converters changed with proton fluence. The exposures were done at LLUMC with 53 $\mathrm{MeV}$ protons. The converter output began to increase near $4.2 \times 10^{10} \mathrm{p} / \mathrm{cm}^{2}$ and by $6.0 \times 10^{10}$ the output had nearly doubled for both parts. For fluence $>6.0 \times 10^{10} \mathrm{p} / \mathrm{cm}^{2}$ the part was current limited.

A detailed analysis of the proton and neutron-induced displacement damage degradation of the converters and the optocouplers it utilizes ensued after these data were taken and is described in [13]. The result of the study was that the optocoupler CTR was falling below the minimum required value for the device to function. Interpoint replaced the optocoupler on one of the failed devices and the part functioned normally. Interpoint has since changed the manufacture of the optocoupler used in these hybrids. It should be noted that Interpoint manufactures high quality radiation hardened $\mathrm{DC} / \mathrm{DC}$ converters, the MHF+ is a COTS hybrid not intended for use in a radiation environment. 


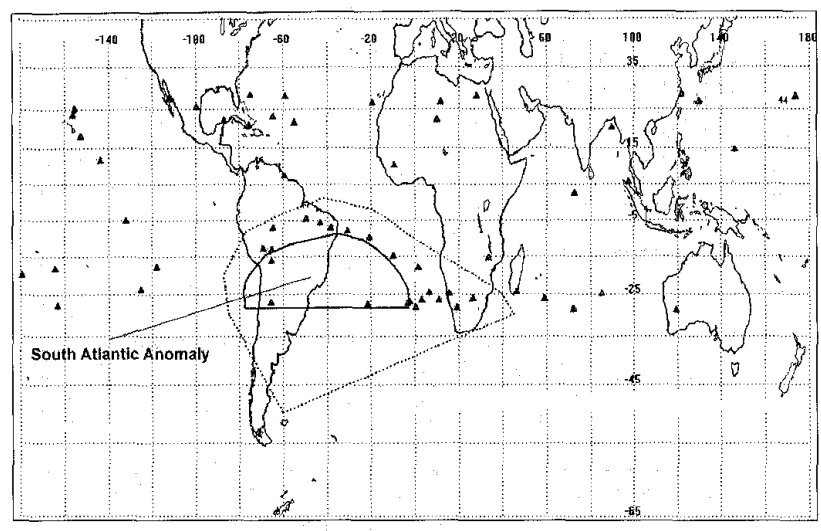

Figure 11. Single Event Transients in optocouplers on the NICMOS instrument flown on the Hubble Space Telescope. SETs in the SAA region occurred before instrument operation was discontinued in that region.

Several issues should be considered when evaluating a device for potential space radiation-induced displacement damage degradation.

- Accurate failure predictions require application specific testing for both the orbit and the circuit configuration.

- Mixed degradation mechanisms of TID and displacement damage make it difficult to quantify the exact amount of degradation that can be attributed to each mechanism.

- Current understanding of NIEL in LED materials is not suitable for accurate rate prediction. Therefore, worst case analysis should be used.

- Co-60 testing does not induce a significant amount of displacement damage. Proton testing must be carried out for each application.

- Careful shielding analysis maybe required because of the increased damage for low energy secondary protons and neutrons.

- Unknown sub-components contained in hybrids could be optocouplers.

- Large uncertainty in the part to part variability in the initial CTR [1].

- Uncertainty in the how normal reliability concerns like lifetime and temperature impacts radiation-induced degradation $[1,6]$.

2) Using Optocouplers that Experience Single Event Transients in a Space Radiation Environment. The space telescope imaging spectograph (STIS) instrument was put into operation on board the Hubble Space Telescope (HST) during the February 1997 servicing mission. Since that time, the instrument electronics have been monitored for SETs due to particle hits on optocouplers. Figure 11 shows recorded SETs on a mercater map. SET rates are so high that the instrument is forced to discontinue operation during HST's passes through the South Atlantic Anomaly (SAA) proton regions. Outside of the SAA, the recorded SETs are due to high energy cosmic rays or solar event particles that penetrate down to the HST circular $600 \mathrm{~km}, 29^{\circ}$ inclination orbit. In 280 days, 14
SETs have occurred on 2 devices outside of the SAA giving an actual SET rate of $2.50 \times 10^{-2}$ per device-day.

Single event transient testing was performed on the optocouplers at three proton facilities. The devices were irradiated at UCD [6]. Irradiations were also carried at ICUF with a $195 \mathrm{MeV}$ proton beam at normal and grazing angle of incidence (results are not given in this paper). Figure 7 gives the results from TRIUMF proton exposures of this optocoupler.

Using parameters derived from the proton measurements above and the geometry assumptions given in [6] for the optocoupler, we estimate the SET rate of $1.5 \times 10^{-2}$ per deviceday using the cosmic ray environment for the HST and the rectangular parallelepiped (RPP) method in CREME96.

This example shows that the dominate mechanism for inducing SETS is not only orbit specific but system operation dependent.

There are several issues that should be considered when evaluating an optocoupler for space radiation-induced SETs.

- Data must be collected using the exact application configuration

- Proton and heavy ion direct ionizations and protoninduced nuclear reactions both cause SETs to occur

- The dominate mechanism will be depend on device type, orbit and potentially system operations

- Rate prediction technique should be the proper combination of the Bendel approach for proton-induced SETs and a modified integral right rectangular parallelepiped (MIRPP) [14 and 6]

\section{CONCLUSIONS}

Evaluation of optocouplers for radiation effects is particular challenging because of the construction of the devices. They typically contain two die and a coupling medium. Each die is usually manufactured from different type of semiconductor. Various types of coupling media are used, for example: thick blobs of Si compound, a mound of carbonbound shards of $\mathrm{SiO}_{2}$, thin layer of coupling compound, and air. These simple devices are, from a radiation effects perspective, complex hybrids that have various radiationinduced performance degradations that depend on the construction of the optocoupler and the radiation environment in which it is to be used.

In this paper we presented a review/discussion of displacement damage-induced degradation of the CTR and concluded that light output degradation for certain AlGaAs LEDs is the probable cause for reduced CTR, while for GaAlP sources the photoresponse of the detector was the major mechanism.

We also showed that generic testing of CTR degradation can lead to incorrect predictions of on orbit CTR degradation. An over prediction of degradation will occur if the devices are tested in the active region but used in saturation. The converse is true for testing in saturation and using the optocouplers in the active region. Also, testing at fixed $V_{c e}$ 
may not give results that are representative of the true degradation mode. We showed that for a loaded device, $\mathrm{V}_{\mathrm{ce}}$ will shift to higher values with radiation.

We presented further evidence that single event transients occurring on the output of an a optocoupler can be induced by direct ionization caused by a single proton based on the angular distribution of the cross section data at various energies. This was initially demonstrated in [6] and is supported by work presented on metal-semiconductor-metal (MSM) in this journal [15]. The SETs can be mitigated with circuit filtering, however the cost is reduced bandwidth.

Finally, we discussed utilization of the optocouplers in the space environment. These hybrid devices are complex from a radiation degradation perspective. True performance predictions require detailed knowledge of optocoupler construction, degradation mechanisms for each type of radiation, application specific radiation characterization, and through specification of the radiation environment and system operation. Special attention must be given to the use of NIEL curves to predict mission specific single energy proton equivalent fluences for assessing displacement damage effects.

\section{ACKNOWLEDGEMENTS}

The authors wish to thank Jim Forney for assisting with radiation testing, Kusum Sahu for providing the gamma data on the TI 4N49, and Martha O'Bryan for her support in preparing this manuscript.

\section{REFERENCES}

[1] B.G. Rax, C.I. Lee, A.H. Johnston, C.E. Barnes, "Total Dose and Displacement Damage in Optocouplers", IEEE Trans. on Nuclear Science, Vol. 43, no. 6, pp. 3167-3173, Dec 1996.

[2] A.S. Epstein and P.A. Trimmer, "Radiation Damage and Annealing Effects in Phonton Coupled Isolators," IEEE Trans. on Nuclear Science, vol 19, no. 6, pp. 391, Dec. 1972.

[3] K.J. Soda, C.E. Barnes, and R.A. Kieh1, "The Effect of Gamma Irradiation on Optical Isolators," IEEE Trans. on Nuclear Science, vol 22, pp. 2475, Dec. 1975.

[4] C.E. Barnes and J.J. Wiczer, "Radiation Effects in Optoelectronic Devices," Sandia Report, SAND84-0771, 1984

[5] M.D. D'Ordine, "Proton Displacement Damage in Optocouplers", 1997 IEEE Radiation Effects Workshop Record, IEEE No. 97TH8293, pp. 122-124, 1997
[6] K.A. LaBel, P.W. Marshall, C.J. Marshall, M. D'Ordine, M.A. Carts, G. Lum, H.S. Kim, C.M. Seidleck, T. Powell, R. Abbott, J.L. Barth, E.G. Stassinopoulos, "Proton-Induced Transients in Optocouplers: In-Flight Anomalies, Ground Irradiation Test, Mitigation and Implications, IEEE Trans. on Nuclear Science, Vol. 44, no. 6, pp. 1885-1892, Dec. 1997.

[7] R. Koga, S.H. Penzin, W.R Crain, S.C Moss, S.D. Pinkerton, S.D. LaLumondiere, and M.C. Maher, , "Single Event Upset (SEU) Sensitivity Dependence of Linear Integrated Circuits (ICs) on Bias Conditions," IEEE Trans. on Nuclear Science, vol 44, no. 6, pp. 2325-2332, Dec. 1997.

[8] R. Ecoffet, S. Duzellier, P. Tastet, C. Aicardi, M. LaBrunee, "Observation of Heavy Ion Induce transients in Linear Circuits," 1994 IEEE Radiation Effects Workshop Record, IEEE No. 94TH06841, pp. 72-78, 1994.

[9] C.J. Dale, P.W. Marshall, G.P. Summers, and E.A. Wolicki, "Displacement Damage Equivalent to Dose in Silicon Devices," Appl. Phys. Lett., Vol. 54, No. 5, pp. 451-453, Jan. 1989.

[10] C.J. Dale, P.W. Marshall, B. Cummings, L. Shamey, and A. Delamere, "Spacecraft Displacement Damage Dose Calculations for Shielded CCDs," SPIE Vol. 1656, pp.476-487, 1992.

[11] G.P. Summers, E.A. Burke, M.A. Xapsos, C.J. Dale, P.W. Marshall, and E.L. Petersen, "Displacement Damage in GaAs Structures," IEEE Trans. Nucl. Sci., Vol. NS-35, No. 6, pp. 1221-1226, Dec. 1988.

[12] S.M. Khanna, A. Houdayer, A. Jorio, C. Carlone, M. Parenteau, and J.W. Gerdes, Jr., "Nuclear Radiation Displacement Damage Prediction in Gallium Arsenide through Low Temperature Photoluminesence Measurements,"IEEE Trans. Nucl. Sci., Vol. NS-43, No. 6, pp. 2601-2608, Dec. 1996.

[13] NASA/GSFC Radiation effects homepage, http://flick.gsfc.nasa.gov/radhome.htm

[14] P.W. Marshall, C.J. Dale, M.A. Carts, and K.A. LaBel, "Particle-Induced Bit Errors in High Performance Fiber Optic Data Links for Satellite Data Management," IEEE Trans. Nucl. Sci., Vol. 41, pp. 1958-1965, Dec. 1994.

[15] C.J. Marshall, P.W. Marshall, M.A. Carts, R.A. Reed, K.A. LaBel, "Proton-Induced Transient Effects in a MetalSemiconductor-Metal (MSM) Photodetector for Optical-Based Data Transfer," to be published in IEEE Trans Nucl. Sci., NS-45, No. 6, Dec 1998 